\begin{tabular}{|c|l|}
\hline Title & $\begin{array}{l}\text { A nalysis of W ater Transport inside Hydrophilic Carbon Fiber Micro-Porous Lay ers with High-Performance Operation } \\
\text { in PEFC }\end{array}$ \\
\hline Author(s) & A oyama, Y usuke; Tabe, Y utaka; Nozaki, Ryo; Suzuki, Kengo; Chikahisa, Takemi; Tanuma, Toshihiro \\
\hline Citation & $\begin{array}{l}\text { Journal of the electrochemical society, 165/7), F484-F491 } \\
\text { https://doi.org/40.1149/2.0801807jes }\end{array}$ \\
\hline Issue Date & 2018.05-10 \\
\hline Doc URL & http://hdl.handle.net/2115/71548 \\
\hline Rights(URL) & https://reativecommons.org/icenses/by/4.0/ \\
\hline Type & article \\
\hline File Information & F484.full.pdf \\
\hline
\end{tabular}

Instructions for use 


\title{
Analysis of Water Transport inside Hydrophilic Carbon Fiber
Micro-Porous Layers with High-Performance Operation in PEFC
}

\author{
Yusuke Aoyama, ${ }^{1}$ Yutaka Tabe, $\odot{ }^{1, *, z}$ Ryo Nozaki, ${ }^{1}$ Kengo Suzuki, ${ }^{1}$ Takemi Chikahisa, ${ }^{1, *}$ \\ and Toshihiro Tanuma ${ }^{2, *}$
}

\author{
${ }^{I}$ Division of Energy and Environmental Systems, Graduate School of Engineering, Hokkaido University, Sapporo, \\ Hokkaido 060-8628, Japan \\ ${ }^{2}$ Asahi Glass Co., Ltd. Research Center, Yokohama, Kanagawa 221-8875, Japan
}

\begin{abstract}
Polymer electrolyte membrane fuel cells using a hydrophilic micro-porous layer (MPL) consisting of carbon fiber (CF) and ionomer show better performance than those using conventional hydrophobic MPL with carbon black (CB) under a wide range of humidity conditions. This study investigates the effects of the wettability and structure of the MPL on cell performance and discusses the mechanism for improving cell performance by the hydrophilic CF-MPL at high humidity conditions. The water distribution inside the MPL is evaluated by a freezing method and cryo-SEM observations, and a comparison of the distribution and the cell performance of hydrophilic and hydrophobic MPL cells with various structures is made. The results show that the performance improvement is caused by preventing increases in concentration overvoltage at high current densities; where the fiber structure and thicker layers are important with hydrophilic MPL. In hydrophilic CF-MPL with better performance, liquid water accumulates in pores at the catalyst layer (CL) side, while there is no liquid water at the gas diffusion layer side. These results indicate that the hydrophilic CF-MPL enhances the liquid water transport from the CL due to the hydrophilic properties, and promotes water evaporation due to the large pores in the thick fiber structure.

(C) The Author(s) 2018. Published by ECS. This is an open access article distributed under the terms of the Creative Commons Attribution 4.0 License (CC BY, http://creativecommons.org/licenses/by/4.0/), which permits unrestricted reuse of the work in any medium, provided the original work is properly cited. [DOI: 10.1149/2.0801807jes]

(cc) BY
\end{abstract}

Manuscript submitted March 19, 2018; revised manuscript received April 23, 2018. Published May 10, 2018. This was Paper 1428 presented at the Phoenix, Arizona, Meeting of the Society, October 11-15, 2015.

Polymer electrolyte membrane fuel cells (PEFCs) are expected to play a leading role as next generation power sources for automobiles, portable devices, and residential cogeneration systems (a so-called combined heat and power; CHP). However, water flooding (the blockage of the gas supply to the reaction area by accumulated water) remains an important issue to be solved, because water flooding decreases cell performance at high current densities. Micro-porous layers (MPLs), typically consisting of carbon black and a hydrophobic polymer between the catalyst layer (CL) and the gas diffusion layer (GDL) are well known as important components in the water management of the PEFC. Therefore, many computational and experimental studies have investigated the role of the MPL in the water management of such cells.

One role of MPL is considered to be that the MPL removes water produced at the cathode CL and mitigates water flooding at high current density conditions. ${ }^{1-5}$ Weber et al. and Pasaogullari et al. reported that the MPL reduces the amount of water passing through the cathode side of the gas diffusion layer by increasing the water flow from cathode to anode through the membrane. ${ }^{1,2}$ Gostick et al. and Lu et al. suggested that cracks in the MPL are pathways for the liquid water discharge, and that this results in limiting water access to the GDL inlet face and reduces water saturation in the GDL. ${ }^{3,4}$ Owejan et al. investigated water transport in the MPL by measuring the performance of cells with various types of MPL, and suggested that the primary role of the MPL is preventing condensed water from accumulating near the cathode CL surface. ${ }^{5}$

To visualize the distribution of water in the vicinity of the cathode MPL, the authors have developed the direct observation method termed the freezing method. ${ }^{6,7}$ The freezing method immobilizes the water in the cell as ice forms, and the ice distribution can be identified at high resolutions by a cryo-scanning electron microscope (cryo-SEM) or an optical microscope. The observation of the crosssectional distributions in MPLs have indicated that the produced water passes through the MPL as vapor under usual PEFC operating conditions while it condenses inside the MPL under low cell temperature operation. ${ }^{6}$ Observations of the CL surface indicated that water accumulation at the CL surface increases with increasing current density,

*Electrochemical Society Member.

${ }^{\text {zE} E-m a i l: ~ t a b e @ e n g . h o k u d a i . a c . j p ~}$ and the addition of an MPL could suppress water accumulation at the CL surface as a result of a closer contact with the CL. ${ }^{7}$ The authors also investigated the effect of the MPL/CL interface structure on the cell performance and the water distribution. ${ }^{8}$ The results showed that a membrane electrode assembly (MEA) made by the gas diffusion electrode (GDE) method under wet conditions increases the cell voltage to above that of an MEA made with the decal method, suggesting that a seamless MPL/CL interface without gaps made by the GDE method could be expected to prevent water accumulation and to improve cell performance.

Recently, some studies have applied hydrophilic compounds to the MPL for further improvements in cell performance under wet and dry conditions. $^{9-13}$ Schweiss et al. developed a hydrophilic wicking MPL which contains hydrophilic alumosilicate fibers in the acetylene black and polytetrafluoroethylene (PTFE), and reported that the hydrophilic MPL contributes to enhance the cell performance regardless of the humidity conditions. ${ }^{10}$ Mukundan et al. used neutron radiography to visualize the liquid water distribution in the MEA using an MPL incorporating hydrophilic alumosilicate fibers. ${ }^{11}$ Hirakata et al. investigated the effect on the cell performance of a hydrophilic porous layer added between the hydrophobic MPL and the GDL, and suggested that the hydrophilic layer absorbs water and improves water removal from the CL to the GDL under wet conditions. ${ }^{12}$ Kitahara et al. applied another type of an additional layer, a hydrophilic MPL composed of carbon black, $\mathrm{TiO}_{2}$, and silicone, and reported that the cell performance can be improved under wet and dry conditions. ${ }^{13}$ These approaches investigate the incorporation of hydrophilic materials in hydrophobic MPL, and report the MPL wettability patterns in-plane $e^{9-11}$ and through-plane. ${ }^{12,13}$ Tanuma et al. developed a novel exclusively hydrophilic MPL composed of carbon fibers and hydrophilic ionomer, and reported that cells using this hydrophilic MPL showed better performance than those using hydrophobic MPL under a wide range of humidity conditions. ${ }^{14,15}$ However, the mechanism of how the whole hydrophilic MPL contributes to the improvement of the cell performance is not fully understood.

The present study investigates the effect of MPL wettability and structure on the cell performance under high humidity conditions to evaluate the mechanism for improving cell performance by the whole hydrophilic MPL in wet conditions. Four types of MPLs with different wettabilities, hydrophilic or conventional hydrophobic properties, and different structures consisting of carbon fibers or conventional carbon 

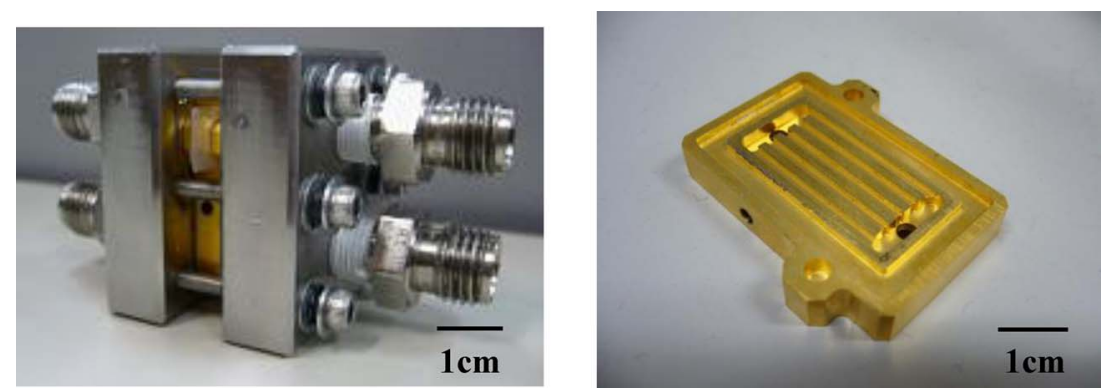

Figure 1. The small single cell used in the investigations here (left) and the bipolar plate with straight channels (right).

Table I. Composition and structure of MPLs used in this study.

\begin{tabular}{lllll} 
& Hydrophobic CB & Hydrophilic CB & Hydrophobic CF & Hydrophilic CF (thinner) \\
\hline Material & Carbon black, PTFE & Carbon black, ionomer & Carbon fiber, PTFE & Carbon fiber, ionomer \\
Amount of solid in MPL coating & $1.0 \mathrm{mg} / \mathrm{cm}^{2}$ & $\leftarrow$ & $3.0 \mathrm{mg} / \mathrm{cm}^{2}$ & $\leftarrow\left(1.0 \mathrm{mg} / \mathrm{cm}^{2}\right)$ \\
MPL thickness & $15 \mu \mathrm{m}$ & $\leftarrow$ & $40 \mu \mathrm{m}$ & $\leftarrow(15 \mu \mathrm{m})$ \\
Pore volume & - & $0.84 \mathrm{~mL} / \mathrm{g}-\mathrm{MPL}^{16}$ & - & $1.33 \mathrm{~mL} / \mathrm{g}-\mathrm{MPL}{ }^{16}$ \\
Mean pore diameter & - & $0.068 \mu \mathrm{m}{ }^{16}$ & - & $0.77 \mu \mathrm{m}{ }^{16}$ \\
Thermal conductivity & $0.15 \mathrm{~W} /(\mathrm{m} \cdot \mathrm{K})$ & $0.20 \mathrm{~W} /(\mathrm{m} \cdot \mathrm{K})$ & - & $0.25 \mathrm{~W} /(\mathrm{m} \cdot \mathrm{K})(0.24 \mathrm{~W} /(\mathrm{m} \cdot \mathrm{K}))$
\end{tabular}

blacks, are used. Further, the water distribution inside the MPL is evaluated by the freezing method and cryo-SEM observations, and the relationship between the water distribution and the cell performance is identified. This paper also discusses the water transport parameters improving the cell performance at high humidity conditions.

\section{Experimental}

Experimental apparatus.-The study used a small single cell with an active area of $1.8 \mathrm{~cm}^{2}(0.9 \mathrm{~cm} \times 2.0 \mathrm{~cm})$ to allow the cell to be frozen and disassembled rapidly with the freezing method. Photos of the cell and the bipolar plate are shown in Fig. 1. The bipolar plate has five straight flow channels and four lands, and is integrated with an electricity collector. The widths of each of the channels and lands were $1.0 \mathrm{~mm}$, and the channel depth was $0.5 \mathrm{~mm}$. The bipolar plates and the membrane electrode assembly (MEA) were enclosed by stainless-steel end-plates.

Four types of cathode MPLs with different wettabilities and structure were used in this study. Table I details the compositions and structures of the MPLs. A schematic view of the relationship between the wettability and the pore diameter of these MPLs is shown in Fig. 2. A commonly used MPL is hydrophobic and composed of carbon black (CB) and PTFE, and this is the 'hydrophobic CB' in Table I and Fig. 2. The $\mathrm{CB}$ is Vulcan $\mathrm{XC}-72 \mathrm{R}$ by Cabot Corporation. The novel hydrophilic MPL developed by Tanuma et al., ${ }^{14,15}$ here termed

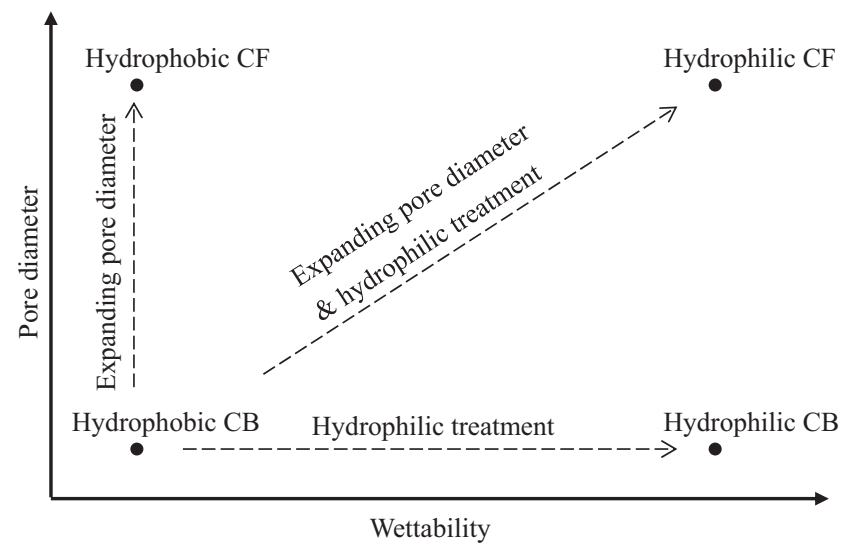

Figure 2. Relationship between the wettability and the pore diameter of the MPL in the investigations here. 'hydrophilic CF', is composed of carbon fiber (CF: VGCF-H, Showa Denko K. K.) and hydrophilic ionomer. Introducing the CF increased the pore diameter of the MPL, and adding the ionomer made the wettability hydrophilic. ${ }^{14,15}$ To investigate the effects of the large pores with the CF and hydrophilic MPL with the ionomer separately, a hydrophilic MPL with the CB (hydrophilic CB) and a hydrophobic MPL with the $\mathrm{CF}$ (hydrophobic $\mathrm{CF}$ ) were also compared. The thickness of the MPL depends on the amount of solid in the MPL slurry. The $\mathrm{CB} / \mathrm{PTFE}$ or the $\mathrm{CB} /$ ionomer slurry with $1.0 \mathrm{mg} / \mathrm{cm}^{2}$ corresponds to the $15 \mu \mathrm{m}$ thick MPL, and the CF/PTFE or the CF/ionomer slurry with $3.0 \mathrm{mg} / \mathrm{cm}^{2}$ corresponds to the $40 \mu \mathrm{m}$ thick MPL. In the later discussion a thinner hydrophilic MPL with the ionomer, shown in brackets in Table I, will be introduced to consider the water transport phenomenon in the hydrophilic CF. The GDL (Improved H2315, hydrophobic treated GDL without an MPL, Freudenberg-NOK) was used for all types of the MPLs. The interface between the cathode side MPL and the CL was fabricated by the gas diffusion electrode (GDE) method. The GDE method forms seamless interfaces without gaps by directly coating catalyst ink on the cathode MPL surface, and prevents water accumulation at the interface between the MPL and the CL. ${ }^{8}$ For the anode side, the hydrophobic CB-MPL was used, and the interface with the anode CL was made by the conventional decal transfer method. These MEAs were experimentally produced by Asahi Glass Co., Ltd., ${ }^{14,15}$ and a detailed characterization of the GDL/MPL was reported in Ref. 16.

Figure 3 shows SEM images of cross-sections of the MPLs for the whole thickness from the MPL/CL to the MPL/GDL interfaces with the hydrophilic CB-MPL (left) and the hydrophilic CF-MPL (right). The structure of the hydrophilic CB-MPL is similar to that of the conventional hydrophobic CB-MPL shown in Ref. 6. The hydrophilic CF-MPL is thicker and the inner structure is clearly different from the MPL with $\mathrm{CB}$, and the pore sizes provided by the $\mathrm{CF}$ are much larger than those of the CB. The appearance of the hydrophobic CF-MPL (not shown here) was similar to the hydrophilic CF-MPL as well as to the two types of the MPLs with $\mathrm{CB}$, and the effects of wettability on the MPL structure appear to be negligible in the experiments in this study.

Experimental methods.- In this study, polarization curves, time series changes in the voltages under constant current density operation, and oxygen gains were measured to investigate the cell performance. The cell temperature and the bubbler temperature were $70^{\circ} \mathrm{C}$ ( $100 \%$ relative humidity $(\mathrm{RH})$ ) with the cell in a thermostatic chamber (HITACHI, EC-25MTP), and the cell temperature was controlled by the chamber temperature. The flow rates of the anode hydrogen and 
(a) CB-MPL

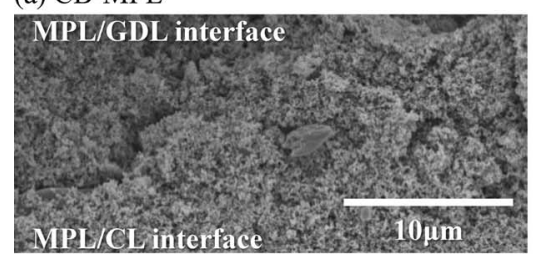

(b) CF-MPL

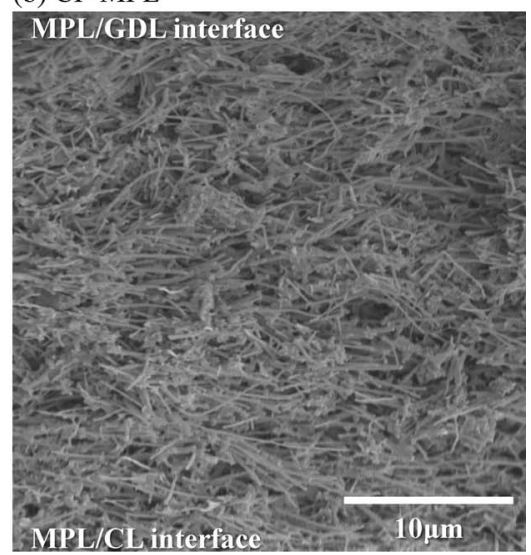

Figure 3. SEM images of cross-sections of the MPLs for the whole thickness from the MPL/CL to the MPL/GDL interfaces, (a) the hydrophilic CB-MPL and (b) the hydrophilic CF-MPL. the cathode air or oxygen were set to 100 and 400 standard cc/min (SCCM, 1.00 standard $\mathrm{cc} / \mathrm{min}=1.67 \times 10^{-8} \mathrm{~m}^{3} / \mathrm{s}$ ), respectively. The stoichiometric ratios of anode hydrogen and cathode air are 11 and 19 at $0.7 \mathrm{~A} / \mathrm{cm}^{2}$, respectively. The cell resistance was measured by an alternating impedance meter at $1 \mathrm{kHz}$ (TSURUGA, model 3566), and the cell voltage, resistance, and temperatures (cell, supply gases, and bubbler) were recorded. In the polarization curve measurements, the current density was maintained for 2 minutes at each measurement point, to reach a steady state, and the cell was considered to have shut down if the cell voltage fluctuated widely and was not stabilized within the 2 minutes. The constant current density operation was carried out at $0.7 \mathrm{~A} / \mathrm{cm}^{2}$ for 1 hour. The oxygen gain was calculated as the difference in polarization curves between the air and oxygen operation, when the cell voltages were corrected for the ohmic loss.

The freezing method and cryo-SEM were used for the visualization of the water distributions in the vicinity of the cathode side MPL. ${ }^{6}$ The freezing method immobilizes the water as ice by rapidly freezing the cell immediately after discontinuing operation, and the cryo-SEM observations make it possible to visualize the water distribution in the vicinity of the MPL at high spatial resolutions without the ice melting. The freezing method consisted of the following steps: first, after achieving constant current density operation at $0.7 \mathrm{~A} / \mathrm{cm}^{2}$, the cell was shut down and immediately cooled at $-40^{\circ} \mathrm{C}$ in the thermostatic chamber to immobilize the water where it is at the instant of the shutdown. In a separate experiment, the authors have confirmed that the water moves little during the cooling process. ${ }^{17}$ It was also confirmed that ice is not generated during freezing when there is no condensed water before cooling, even if the MEA is hydrated. Next, the cell was disassembled in the thermostatic chamber at $-40^{\circ} \mathrm{C}$, and the MEA was cooled further in liquid nitrogen to prevent sublimation of the frozen water. Then, the MEA was cut into three or four pieces and set in the sample holder in liquid nitrogen. Finally, the sample was moved to the cryo-SEM (JEOL, JSM-6701F with Gatan, ALTO2500) and observed at $-150^{\circ} \mathrm{C}$ with an acceleration voltage of $5 \mathrm{kV}$.

\section{Results and Discussion}

Effect of the MPL structure and wettability on the cell performance.-Figure $4 \mathrm{a}$ shows the polarization curves and resistance of the cells using the hydrophilic CF-MPL and the conventional hydrophobic CB-MPL at the cathode side. The voltage of the hydrophilic CF-MPL is higher than that of the hydrophobic CB-MPL under high current density conditions. This is consistent with the results reported by Tanuma et al. using a cell with an active area of 25 $\mathrm{cm}^{2}$ in wet conditions $\left(80^{\circ} \mathrm{C}, 100 \% \mathrm{RH}\right),{ }^{18}$ supporting the assumption that the hydrophilic CF-MPL shows the better performance. It has also been confirmed that the cell performance with the hydrophilic $\mathrm{CF}-\mathrm{MPL}$ is improved in drier conditions $\left(70^{\circ} \mathrm{C}, 50 \% \mathrm{RH},{ }^{19}\right.$ and $80^{\circ} \mathrm{C}$, $30 \% \mathrm{RH}) .{ }^{18}$ In this study, the investigations are focused on the phenomena under flooded conditions. The difference in the cell voltages becomes larger as the current density increases, resulting in significant differences in the limiting current density when the voltage drops to around $0.2 \mathrm{~V}$. There are no significant differences in the resistance of these cells, and this suggests that the voltage difference is little affected by the ohmic resistance loss. Figure $4 \mathrm{~b}$ shows the changes in the cell voltage and the cell resistance for 1 hour of constant current density operation at $0.7 \mathrm{~A} / \mathrm{cm}^{2}$ for both the cells with the hydrophilic CF-MPL and hydrophobic CB-MPL. The voltages of the hydrophilic CF-MPL are higher during the operation, as also suggested by the results in Fig. 4a. The values of the resistance for these cells are similar, while there is some irregularity in the cell resistance of the hydrophobic CB-MPL which may be caused by flooding.

To investigate factors that would be effective in improving the cell performance by the hydrophilic CF-MPL, cells using the hydrophilic CB-MPL and hydrophobic CF-MPL at the cathode side were also compared with a cell using the conventional hydrophobic CB-MPL. The hydrophobic and hydrophilic CB-MPLs had similar structures and different wettabilities; the hydrophobic CF-MPL had similar wettability to the hydrophobic CB-MPL but a different structure with the larger pore diameters. Figure 5a shows the polarization curves (a)

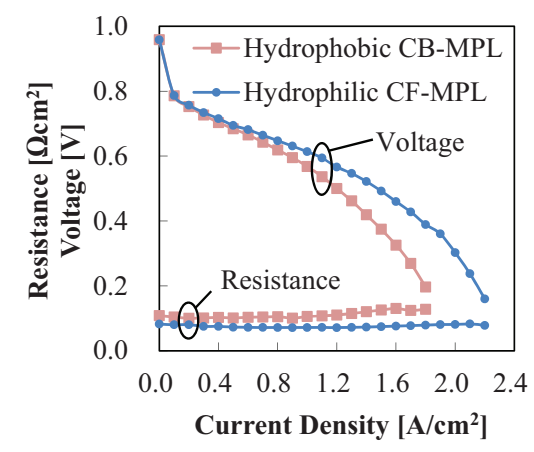

(b)

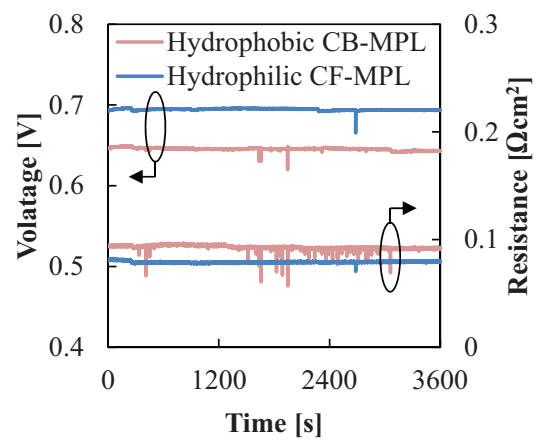

Figure 4. Plots of (a) polarization curves and cell resistances, and (b) changes in the cell voltage and resistance at the constant current density of $0.7 \mathrm{~A} / \mathrm{cm}^{2}$ with the hydrophilic CF-MPL and the hydrophobic CB-MPL. 
(a)

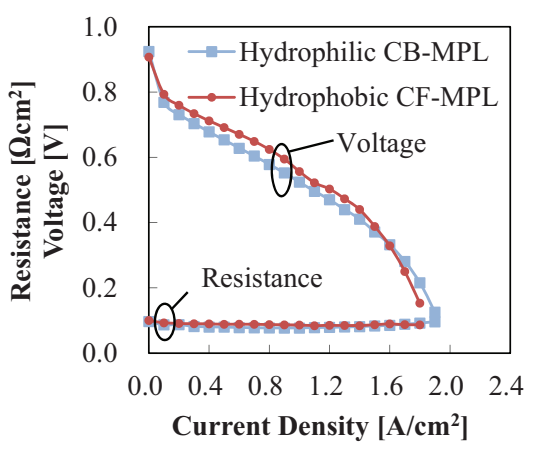

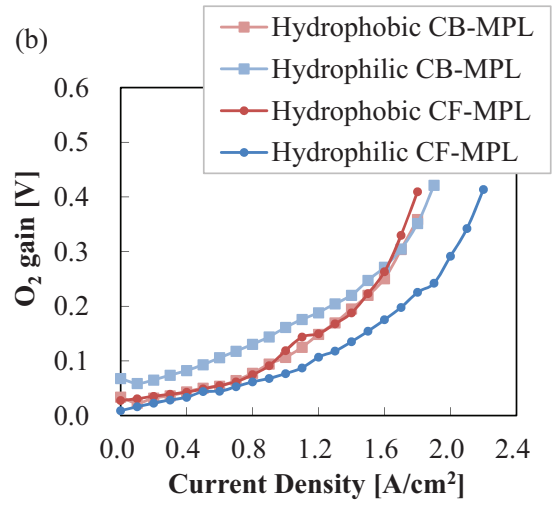

Figure 5. (a) Polarization curves and cell resistances of the hydrophilic CB-MPL and the hydrophobic CF-MPL, and (b) plot of the oxygen gains of the four types of MPLs. and resistances of these cells with the hydrophilic CB-MPL and the hydrophobic CF-MPL. Overall, the performances are similar to those of the hydrophobic CB-MPL in Fig. 4a. There is a minor difference in that the voltages of the hydrophilic CB-MPL become lower at low current densities from around $0.4 \mathrm{~A} / \mathrm{cm}^{2}$, while the limiting current density is slightly higher. This could suggest that the hydrophilic MPL with a conventional CB structure is more easily affected by water accumulation, but the effect is less likely to become a serious impediment than with the hydrophobic MPLs. From the comparison of the performance of the cells with the four types of MPLs, it may be concluded that the combination of increasing the pore diameter and applying a hydrophilic treatment contributes to the improvement in the cell performance of the hydrophilic CF-MPL, as shown in Fig. 4a.

To further confirm the reasons for the different cell performances, the oxygen gains of the cells with the four types of cathode MPLs in Figs. $4 \mathrm{a}$ and $5 \mathrm{a}$ were compared. The oxygen gain indicates the oxygen transport resistance in the cell, and a larger oxygen gain means a higher concentration overvoltage. Figure $5 \mathrm{~b}$ shows the oxygen gains of the hydrophobic CB-MPL, the hydrophilic CB-MPL, the hydrophobic CF-MPL, and the hydrophilic CF-MPL. The oxygen gain of the hydrophilic CF-MPL is the lowest among the four types of MPLs, suggesting that the cell with the hydrophilic CF-MPL is less affected by the concentration overvoltage. Because the experimental condition was wet, the concentration overvoltage is considered to be caused by water flooding, and the result indicates that the hydrophilic CF-MPL is the more effective to suppress an increase in the concentration overvoltage under severely flooded conditions. The oxygen gains of the other MPLs in Fig. 5b also correspond to differences suggested by the characteristics of the polarization curves in Figs. 4a and 5a. The oxygen gains of the hydrophilic CB-MPL are higher than those of the hydrophobic CB-MPL and the hydrophobic CF-MPL even at the low current densities around $0.4 \mathrm{~A} / \mathrm{cm}^{2}$, and becomes lower (than the two hydrophobic MPL cells) at current densities above $1.6 \mathrm{~A} / \mathrm{cm}^{2}$. This would indicate that the treatment to make the porous layer hydrophilic by adding hydrophilic ionomer has the potential to improve the cell performance under flooded conditions, but that an appropriate structure such as large pore diameters like those formed with the $\mathrm{CF}$ is critical to preventing an increase in the concentration overvoltage which arises even in low current density conditions.

Ice distributions in the four types of the MPLs.-To evaluate the water transport inside the MPL and its effect on the cell performance, the water distribution in the cells was observed by the freezing method and cryo-SEM. Figure 6 shows cryo-SEM images inside (a) the (a) Hydrophobic CB-MPL at CL side

Before operation
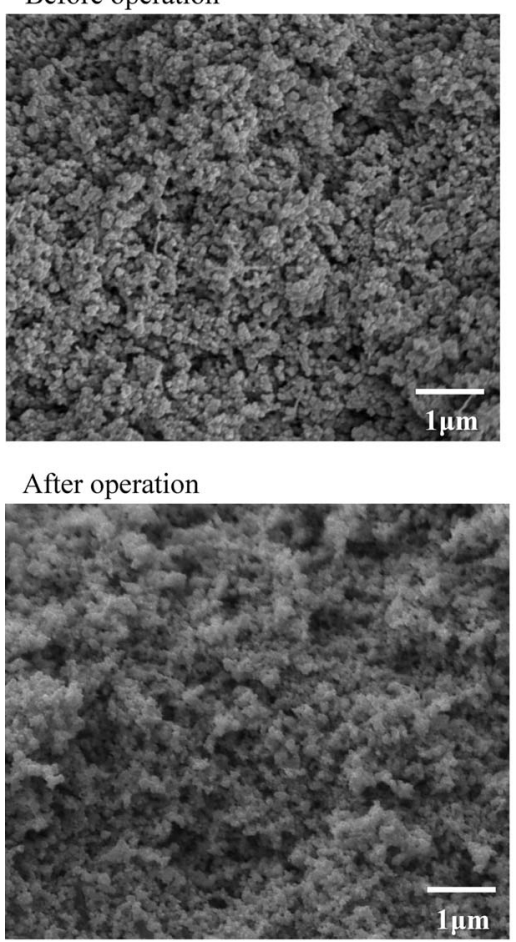

(b) Hydrophilic CB-MPL at CL side

\section{Before operation}

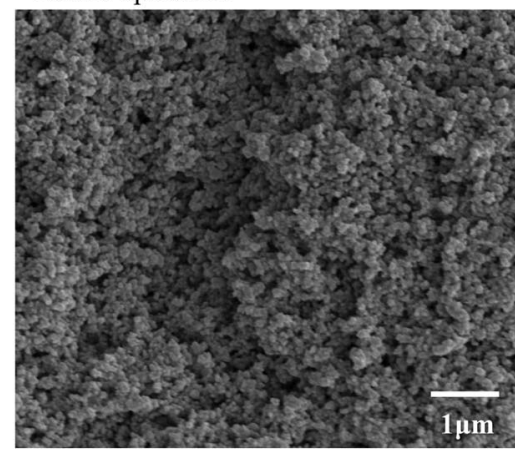

After operation

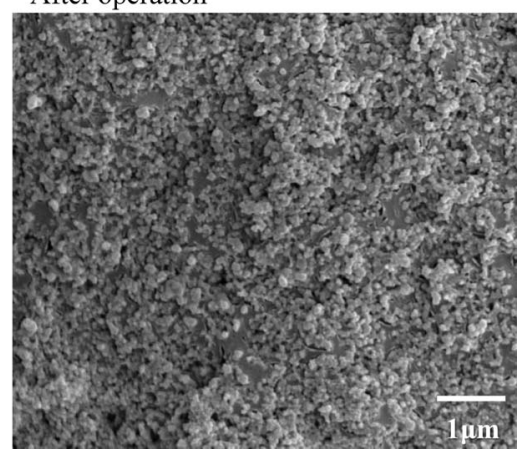

Figure 6. Cryo-SEM images of cross-sections of (a) the hydrophobic CB-MPL and (b) the hydrophilic CB-MPL before and after operating the cell at $0.7 \mathrm{~A} / \mathrm{cm}^{2}$ for 1 hour. 
(a) Whole CF-MPL

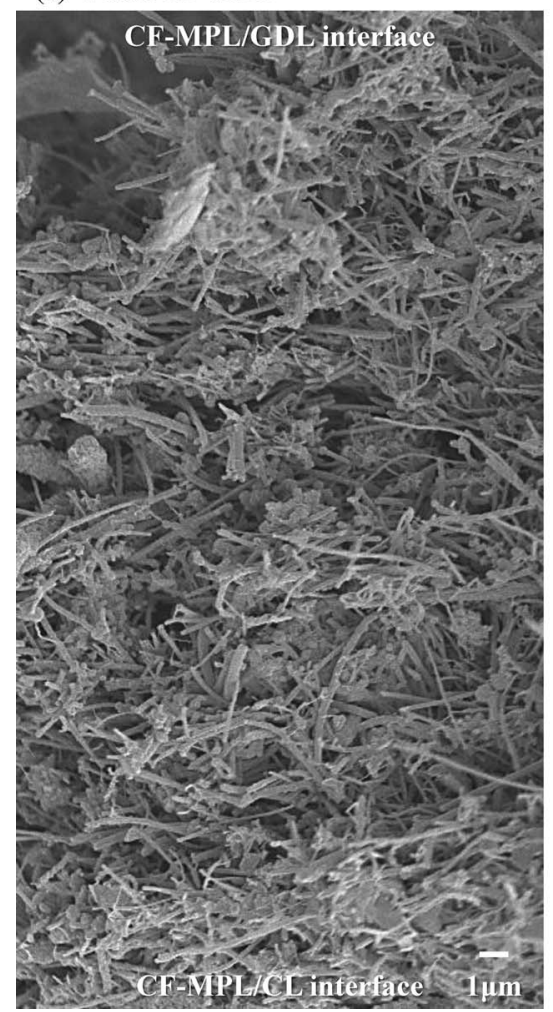

(b) CF-MPL at GDL side

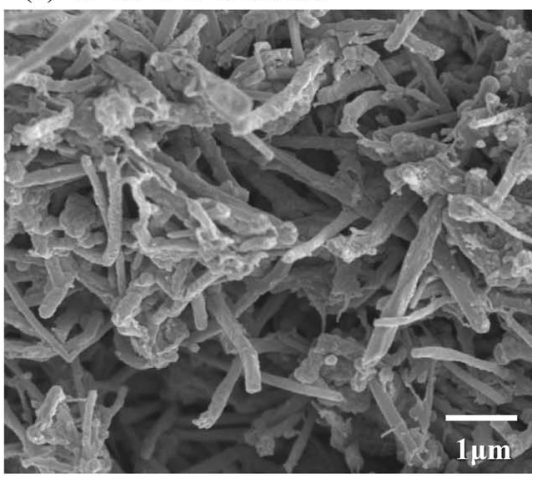

(c) CF-MPL at CL side

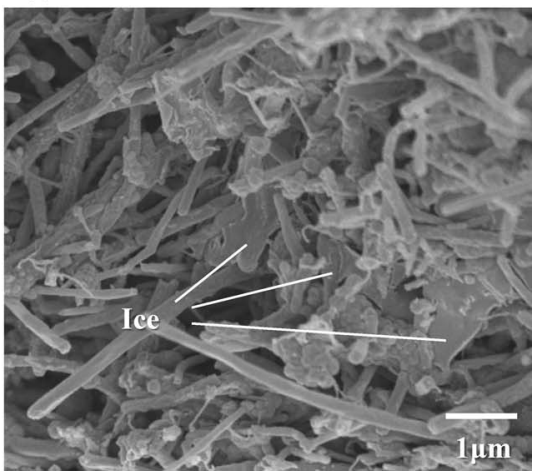

Figure 7. Cryo-SEM images of cross-sections of the hydrophobic CF-MPL for (a) the whole thickness, (b) for the GDL side, and (c) for the CL side after operating the cell at $0.7 \mathrm{~A} / \mathrm{cm}^{2}$ for 1 hour. hydrophobic CB-MPL and (b) the hydrophilic CB-MPL at the cathode side before and after operating the cell at $0.7 \mathrm{~A} / \mathrm{cm}^{2}$ for 1 hour. The areas of the MPL images are near the catalyst layer (CL) interfaces. These images showing the CL structure and ice distribution were quite uniform for all of the MPLs. Similar images were also obtained with all of the observations of both the MPLs at various in-plane locations. Inside the hydrophobic CB-MPL after the operation of Fig. 6a, there is no ice observed with the porous structure, similar to the situation before the start of operation. This is similar to the appearance observed under a little drier condition $\left(70^{\circ} \mathrm{C}, 81 \% \mathrm{RH}\right),{ }^{8}$ suggesting that the produced water passes through the MPL in vapor form. This would be because the water vapor transport capacity driven by the saturation pressure gradient due to the temperature gradient, which was proposed by Owejan et al., ${ }^{5}$ is sufficient to maintain unhindered passage under ordinary operating conditions. ${ }^{8}$ In Fig. 6 b, inside the hydrophilic CB-MPL after the operation there is ice filling some pores with some pores remaining vacant. As mentioned above, the water vapor transport capacity of the MPL can be estimated to be sufficient at $70^{\circ} \mathrm{C}$, and it may be considered that the observed ice corresponds to the water transported from the CL to the MPL in liquid form and due to the hydrophilic property. This liquid water may cause the increase in the concentration overvoltage of the hydrophilic CB-MPL from the low current density in Fig. 5b.

Figure 7 shows the cryo-SEM images inside the hydrophobic CFMPL at the cathode side after operating the cell at $0.7 \mathrm{~A} / \mathrm{cm}^{2}$ for 1 hour. Figure 7 a shows the whole thickness of the hydrophobic CFMPL, and Figs. $7 \mathrm{~b}$ and $7 \mathrm{c}$ are the magnified images of the MPL at the GDL and the CL sides. The top of Figs. $7 \mathrm{a}$ and $7 \mathrm{~b}$ correspond to the MPL/GDL interface, and the bottom of Figs. 7a and 7c correspond to the MPL/CL interface. Generally there is no ice observed in the larger pores composed of the $\mathrm{CF}$; and there is some ice scattered on/among the fibers near the MPL/CL interface as also shown in Fig. 7c. This could be due to the larger diameter allowing liquid water to penetrate into the hydrophobic pores. Additionally, large ice accumulations were formed at the port of the MPL/CL interface (not shown in Fig. 7), suggesting that there are larger spaces at the interface between the MPL and the CL for water to accumulate ${ }^{7}$ even when the interface was formed by the gas diffusion electrode (GDE) method.

The hydrophilic CF-MPL shows the better performance with lower concentration overvoltages in Figs. 4 and 5. Figure 8 shows the cryoSEM images of the hydrophilic CF-MPL at the cathode side after operating the cell at $0.7 \mathrm{~A} / \mathrm{cm}^{2}$ for 1 hour: (a) image across the whole thickness of the hydrophilic CF, and magnified images of the MPL at (b) for the GDL and (c) for the CL sides. The ice distribution inside the hydrophilic CF-MPL is uneven in the through-plane direction of the MPL. An interface between pores where ice was observed and empty pores (enclosed by a circle) is clearly shown in Fig. 8a. The pores of the MPL at the CL side appear to be filled with large amounts of ice (Fig. 8c), while there is no ice at the GDL side (Fig. 8b). This ice distribution was observed at all of the observation points, while the interface between the ice and the pores varied somewhat in location. The differences in the changes of the images without the interface of the pores with ice and the pores without ice was not confirmed by all observations at various in-plane locations, indicating that the layers having pores with ice near the CL occupy the large part of the MPL. In Fig. 8c, there is sheet of ice in almost all of the pores, and this is similar to the ice distribution observed previously in the hydrophobic CB-MPL at the low temperature conditions of $5^{\circ} \mathrm{C} .^{8}$ Although the cell voltages of the hydrophobic CB-MPL at $5^{\circ} \mathrm{C}$ becomes lower, ${ }^{8}$ the cell performance with the hydrophilic CF-MPL at $70^{\circ} \mathrm{C}$ is improved as shown in Fig. 4a, even with sheets of ice present as in Figs. 8a and 8c. This difference in the effect of observed ice on the performance cannot be explained by the cryo-SEM analyses here, because of the difficulties of making a quantitative evaluation of the amounts and the structure of the ice in the MPL from the images. There may be paths, which it was impossible to identify, but which transport oxygen remaining inside the MPL, and a possible scheme for the water transport will be discussed below.

To discuss the water transport in the hydrophilic CF-MPL which provided the uneven ice distribution in Fig. 8, cyro-SEM observations with a higher current density was also conducted. Figure 9 shows 
(a) Whole CF-MPL

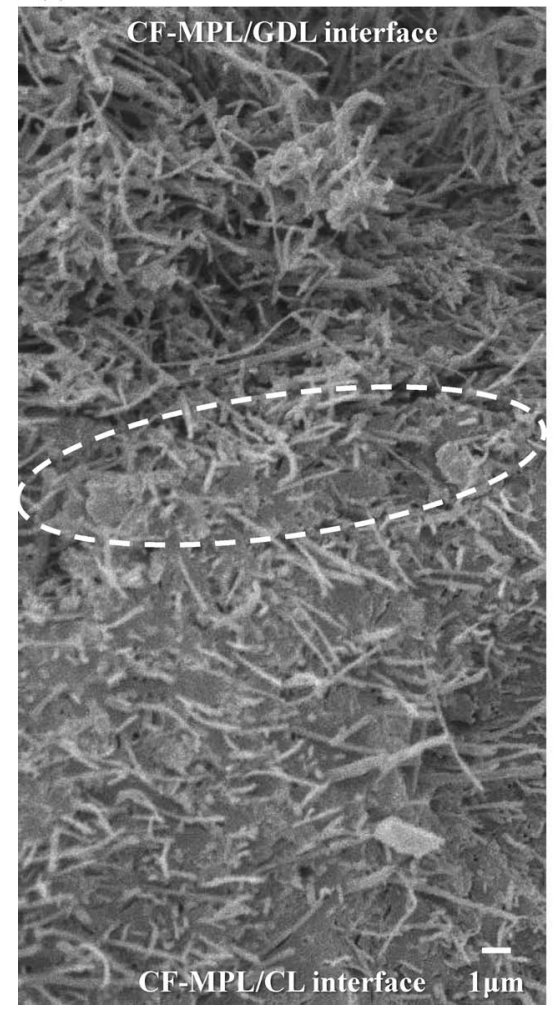

(b) CF-MPL at GDL side

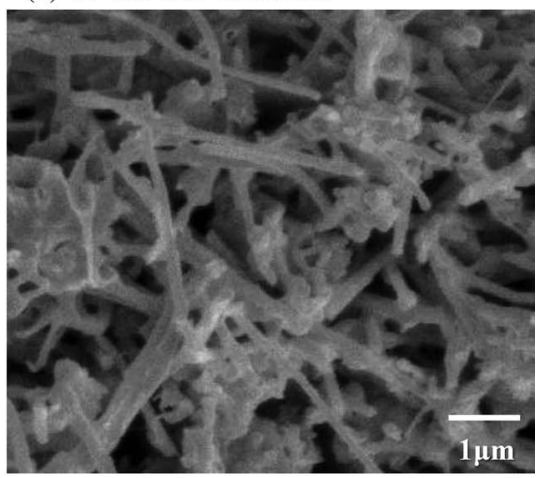

(c) CF-MPL at CL side

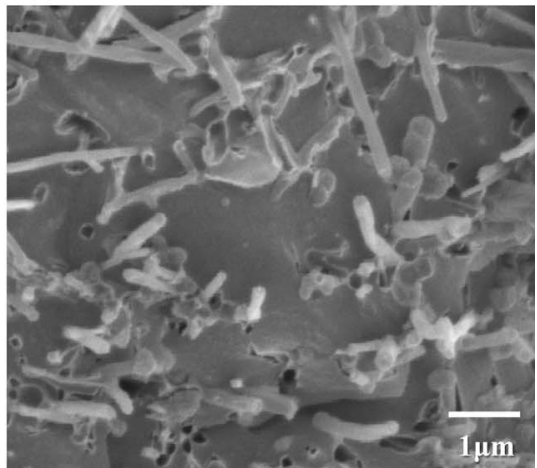

Figure 8. Cryo-SEM images of cross-sections of the hydrophilic CF-MPL with better performance for (a) the whole thickness, (b) the GDL side, and (c) the CL side after operating the cell at $0.7 \mathrm{~A} / \mathrm{cm}^{2}$ for 1 hour. images of the hydrophilic CF-MPL after operation at $1.4 \mathrm{~A} / \mathrm{cm}^{2}$ for 1 hour. The region where ice was observed here has spread further toward the GDL side of the MPL in the image showing the whole thickness (Fig. 9a). At the GDL side, there are parts where the pores were filled with large amounts of ice as shown in Fig. 9b, and pores not filled with ice remained in some regions (not shown here). The ice distribution at the CL side (Fig. 9c) is similar to that at the lower current density (Fig. 8c). It should be noted that the cell voltage with the hydrophilic CF-MPL is also maintained higher than the cells with the other MPLs during this higher current density operation. (a) Whole CF-MPL

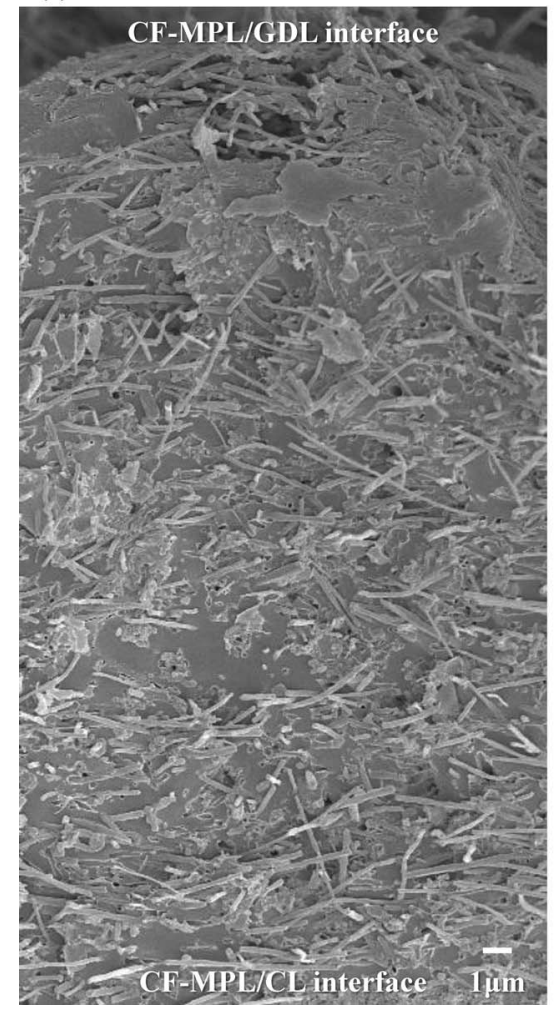

(b) CF-MPL at GDL side

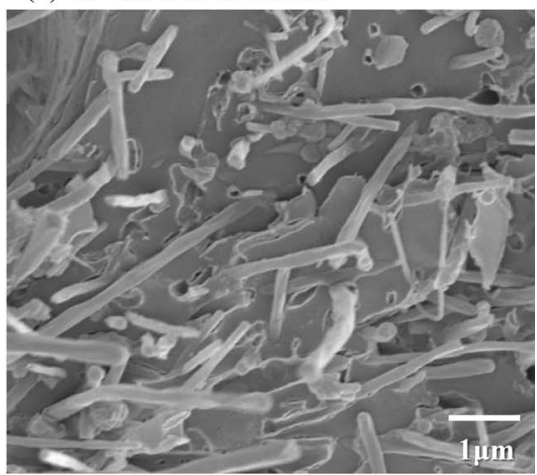

(c) CF-MPL at CL side

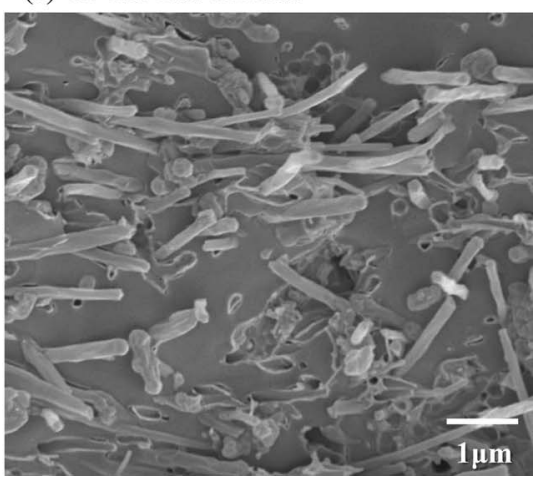

Figure 9. The effect of the operating current density on the ice distribution in the hydrophilic CF-MPL; cryo-SEM images after operating the cell at $1.4 \mathrm{~A} / \mathrm{cm}^{2}$ for 1 hour for (a) the whole thickness, (b) the GDL side, and (c) the CL side. 
(a) $0.7 \mathrm{~A} / \mathrm{cm}^{2}$

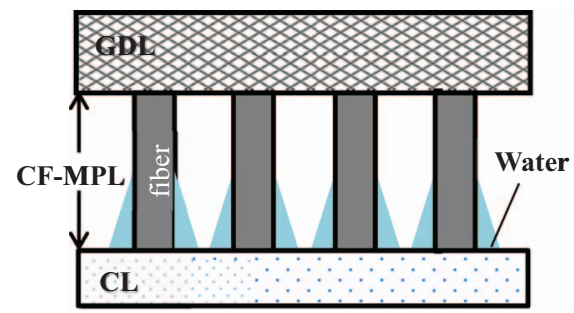

(a)

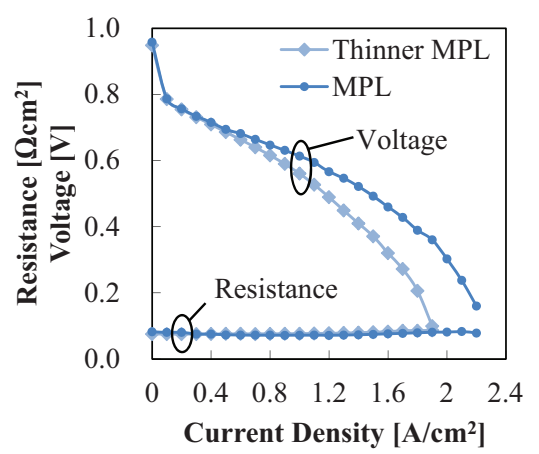

(b) $1.4 \mathrm{~A} / \mathrm{cm}^{2}$

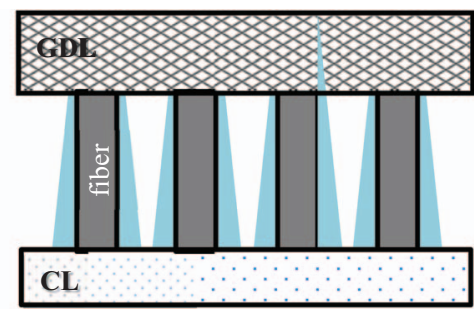

(b)

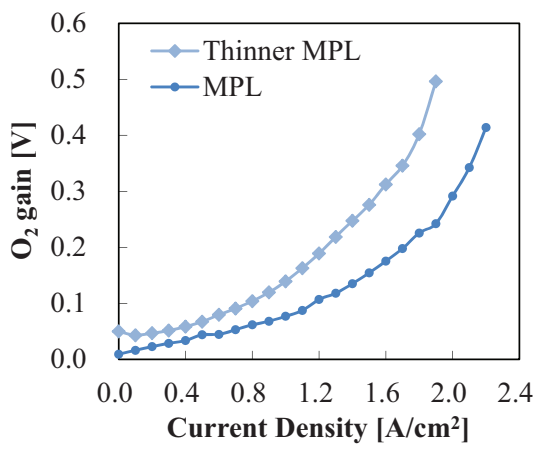

Figure 10. Schematic cross-sectional diagrams of the experimentally suggested water transport in the hydrophilic CF-MPL at (a) $0.7 \mathrm{~A} / \mathrm{cm}^{2}$ and (b) $1.4 \mathrm{~A} / \mathrm{cm}^{2}$. The hydrophilic CF-MPL draws up water from the CL due to the hydrophilic properties with water spreading across the CF surface, promoting evaporation of the water due to the fiber structure with the large pores.

Figure 11. (a) Polarization curves and cell resistance, and (b) oxygen gains of the thinner hydrophilic CF-MPL, plotted with the hydrophobic CF-MPL in Figs. $4 \mathrm{a}$ and $5 \mathrm{~b}$.
Water transport and performance improvement of the MPL with the hydrophilic $\boldsymbol{C F}$. - The possible water transport in the hydrophilic CF-MPL, suggested by the experimental results of Figs. 4, 5b, 8, and 9, is illustrated in schematic cross-sectional diagrams in Fig. 10. The fiber orientations are expressed only in the thickness direction for ease of description. Here, we wish to hypothesize that the improvement in the cell performance with the hydrophilic CF-MPL is caused by a high ability to transport liquid water from the CL and to evaporate it in the MPL under the wet conditions in this study. The hydrophilic CF-MPL draws up water from the CL due to the hydrophilic property with water spreading on the CF surface, as shown in Fig. 10. The fiber structure with the large pores may maintain the water as a film without the pores becoming completely filled, increase the surface area of the water film on the $\mathrm{CF}$, and then the hydrophilic CF-MPL is able to promote evaporation of the water. In the operation at $0.7 \mathrm{~A} / \mathrm{cm}^{2}$, the rate of spreading of the produced water on the CF surface is balanced with the rate of evaporation of the water film, and a steady water distribution is maintained as suggested in Fig. 10a. As the current density increases, the rate of water production becomes higher, and the water film spreads to the GDL side to increase the surface area with a concomitant higher evaporation rate of the total water present in the MPL, as shown in Fig. 10b. These situations in Figs. 10a and $10 \mathrm{~b}$ correspond to the images in Figs. 8 and 9, although it is difficult to identify the structure of the water film from the observed images. The hypothesis proposed here and the measured results in Figs. 4 and $5 b$ suggest the possibility that there are sufficient open paths to ensure the passage of sufficient oxygen as long as a sufficient evaporation rate is achieved with the large surface area of the water film in the pores.

The above discussion suggests that an adequate thickness of the MPL with the hydrophilic CF is necessary to increase the surface area of the water film till the high current density condition is reached. To validate this suggestion, the performance and ice distribution with a thinner hydrophilic CF-MPL were investigated. Rather than the commonly and so far used $40 \mu \mathrm{m}$ thick CF-MPL, a thinner CF-MPL film of about $15 \mu \mathrm{m}$ was used, as shown in Table I. Figure 11 plots (a) the polarization curves and resistances and (b) the oxygen gains of the cells using the thinner and normally thick hydrophilic CF-MPLs (thinner MPL and MPL respectively in Fig. 11). The results for the thicker hydrophilic CF-MPL are the same as those in Figs. $4 \mathrm{a}$ and $5 \mathrm{~b}$. As expected, the voltages of the thinner MPL are lower than those of the thicker MPL. The oxygen gains of the thinner MPL are also higher, supporting the above suggestion: the tolerance to flooding by the thinner hydrophilic CF-MPL is insufficient even with the larger pore size of the MPL provided by the fiber structure.

Figure 12 shows a cryo-SEM image of the whole thickness of the thinner hydrophilic CF-MPL after operating the cell at $0.7 \mathrm{~A} / \mathrm{cm}^{2}$ for 1 hour. Large amounts of ice are apparent in the pores, and the ice distributes uniformly inside the MPL from the CL to the GDL sides. This is considered to be because the thickness of the thinner hydrophilic CF-MPL is inadequate to evaporate all of the water, and the result is the growth of the water film on the CF and blockage of the oxygen supply through the MPL. In the case of the hydrophilic CB-MPL after the operation in Fig. 6b, ice also distributed uniformly from the CL to the GDL sides. This indicates that only inadequate amounts of water evaporate, something that may be ascribed to the inadequate pore size of the MPL composed of the CB preventing the formation of a water film on the CB surface with the large surface area for the higher evaporation rate of water. It may be concluded that the hydrophilic CF-MPL provides the better ability to evaporate water due to the fiber structure and adequate thickness of the MPL, and that it acts as a barrier to prevent flooding at high current densities. Detailed analyses of the distribution and structure of the water in the hydrophilic CF-MPL are necessary to validate the hypothesis suggested here.

Whole thinner CF-MPL

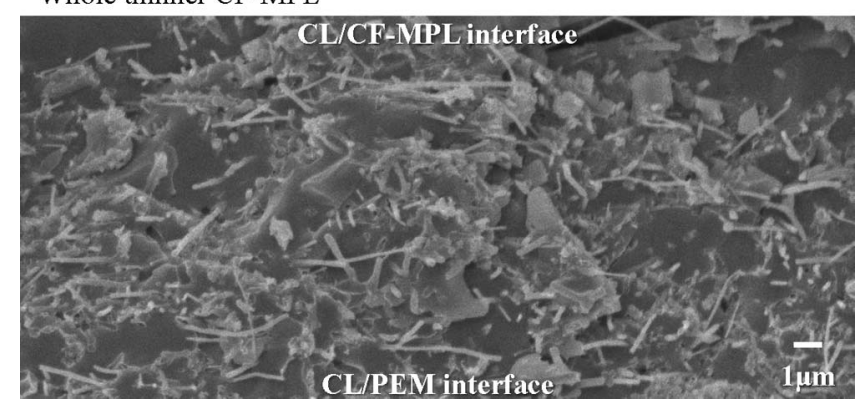

Figure 12. Cryo-SEM image of the whole thickness of the thinner hydrophilic CF-MPL after operating the cell at $0.7 \mathrm{~A} / \mathrm{cm}^{2}$ for 1 hour. 
The above results indicate that the improvement in the cell performance using the hydrophilic CF-MPL is caused by a decrease in the amount of water inside the CL. However, an accurate numerical comparison of the amounts of ice in the CLs was difficult using the cryo-SEM observations and there were no notable differences among the CL images (not shown here). This shows the difficulty associated with performing a quantitative analyses of the ice amounts from the images, and further investigation is needed to accurately elucidate the water transport phenomena in the CL. Recently, the authors have developed the limiting current analysis ${ }^{20,21}$ to apply to flooded conditions..$^{22}$ In that analysis the total oxygen transport resistance in the cell, which is affected by the liquid water accumulated in the cell, is divided into two resistances: the resistance dependent on the total pressure of the cathode gas and that independent of the total pressure. The pressure independent resistance can be assumed to correspond approximately to the oxygen transport resistance in the CL, the Knudsen diffusion resistance in the pores of the $\mathrm{CL}$, and the transport resistance from the gas in the pores to the Pt surface through the ionomer, and this allows the oxygen transport resistance in the CL to be evaluated quantitatively. The results suggest that the oxygen transport resistance in the CL with the hydrophilic CF-MPL is maintained lower than that with the hydrophobic CB-MPL at high current densities, ${ }^{22}$ supporting the experimentally suggested water transport illustrated in Fig. 10 that the hydrophilic CF-MPL promotes the draining of water from the CL to the MPL. Although the above results are limited to a specific condition and further detailed analyses such as the limiting current ${ }^{22}$ and oxygen gain ${ }^{23}$ analyses are necessary for a more precise evaluation of the oxygen transport resistance in the CL, it may be concluded that the hydrophilic CF-MPL enhances the transport of liquid water in the CL due to the hydrophilic fibers. The MPL structure with the large pores and sufficient thickness provides a superior environment to evaporate the water in the MPL, and this results in the better anti-flooding performance at high current densities, as shown in the comparison of the cell performances (Figs. 4, 5 and 11).

\section{Conclusions}

This paper investigates the mechanism for improving the cell performance by the MPL with a hydrophilic CF based on observations of the water distribution in the vicinity of the cathode side MPL. The major conclusions may be summarized as follows:

1. The cell using the hydrophilic CF-MPL shows higher cell voltages than those using the MPL with the conventional hydrophobic CB-MPL at high current densities. The oxygen gains of the hydrophilic CF-MPL are also lower, and these results suggest that the hydrophilic CF-MPL prevents increases in the concentration overvoltage due to flooding under higher current density conditions. The increase in the cell voltage and the decrease in the oxygen gain are not observed in the cells using the hydrophilic CB-MPL and the hydrophobic CF-MPL in this study.

2. The freezing method and the cryo-SEM observations show that ice distribution inside the hydrophilic CF-MPL is uneven in the through-plane direction of the MPL after the $70^{\circ} \mathrm{C}$ operation at the current density of $0.7 \mathrm{~A} / \mathrm{cm}^{2}$. This suggests that liquid water remains in the pores of the hydrophilic CF-MPL at the CL side, while there is no water at the gas diffusion layer GDL side. At 1.4 $\mathrm{A} / \mathrm{cm}^{2}$, the region where ice was observed expands to the GDL side of the MPL, where a sufficient porosity remains and allows the transport of oxygen through the MPL.
3. From the measured cell performance and the observed ice distribution, a possible mechanism for the improvements in the cell performance by the hydrophilic CF-MPL is proposed to be that the hydrophilic CF-MPL enhances the transport of liquid water in the CL due to the hydrophilic properties with water able to spread across the CF surface, and promotes evaporation of the water with the large pores and sufficient thickness maintaining the water as a film and increasing the surface area of the water film on the $\mathrm{CF}$.

\section{Acknowledgments}

The authors thank T. Hayashi (an undergraduate student of Hokkaido University) for help with the experiments and the cryoSEM observations. The cryo-SEM observations were conducted at the Electron Microscope Laboratory, Research Faculty of Agriculture, Hokkaido University. This research was supported by JSPS KAKENHI grant Number 261599.

\section{ORCID}

Yusuke Aoyama (D https://orcid.org/0000-0001-9287-7050

Yutaka Tabe (1D https://orcid.org/0000-0002-6160-1526

\section{References}

1. A. Z. Weber and J. Newman, J. Electrochem. Soc., 152, A677 (2005)

2. U. Pasaogullari, C.-Y. Wang, and K. S. Chen, J. Electrochem. Soc., 152, A1574 (2005).

3. J. T. Gostick, M. A. Loannidis, M. W. Fowler, and M. D. Prizker, Electrochem. Commun., 11, 576 (2009).

4. Z. Lu, M. Daino, C. Rath, and S. G. Kandlikar, Int. J. Hydrogen Energy, 35, 4222 (2010).

5. J. P. Owejan, J. E. Owejan, W. Gu, T. A. Trabold, T. W. Tighe, and M. F. Mathias, J. Electrochem. Soc., 157, B1456 (2010).

6. Y. Aoyama, K. Suzuki, Y. Tabe, and T. Chikahisa, Electrochem. Commun., 41, 72 (2014).

7. Y. Tabe, Y. Aoyama, K. Kadowaki, K. Suzuki, and T. Chikahisa, J. Power Sources, 287, 422 (2015)

8. Y. Aoyama, K. Suzuki, Y. Tabe, T. Chikahisa, and T. Tanuma, J. Electrochem. Soc., 163, F359 (2016)

9. X. L. Wang, H. M. Zhang, J. L. Zhang, H. F. Xu, Z. Q. Tian, J. Chen, H. X. Zhong, Y. M. Liang, and B. L. Yi, Electrochim. Acta, 51, 4909 (2006).

10. R. Schweiss, M. Steeb, and P. M. Wilde, FUEL CELLS, 10, 1176 (2010),

11. R. Mukundan, J. Davey, J. D. Fairweather, D. Spernjak, J. S. Spendelow, D. S. Hussey, D. Jacobson, P. Wilde, R. Schweiss, and R. L. Borup, ECS Trans., 33(1), 1109 (2010)

12. S. Hirakata, M. Hara, K. Kakinuma, M. Uchida, D. A. Tryk, H. Uchida, and M. Watanabe, Electrochim. Acta, 120, 240 (2014).

13. T. Kitahara, H. Nakajima, M. Inamoto, and K. Shinto, J. Power Sources, 248, 1256 (2014).

14. T. Tanuma and S. Kinoshita, J. Electrochem. Soc., 159, B150 (2012)

15. T. Tanuma and S. Kinoshita, J. Electrochem. Soc., 161, F94 (2014).

16. T. Tanuma, M. Kawamoto, and S. Kinoshita, J. Electrochem. Soc., 164, F499 (2017).

17. K. S. S. Naing, Y. Tabe, and T. Chikahisa, J. Power Sources, 196, 2584 (2011)

18. T. Tanuma and S. Kinoshita, Energy Procedia, 28, 12 (2012).

19. Y. Aoyama, K. Suzuki, Y. Tabe, T. Chikahisa, and T. Tanuma, ECS Trans., 64(3), 527 (2014).

20. D. R. Baker, D. A. Caulk, K. C. Neyerlin, and M. W. Murphyc, J. Electrochem. Soc., 156, B991 (2009).

21. N. Nonoyama, S. Okazaki, A. Z. Weber, Y. Ikogi, and T. Yoshida, J. Electrochem Soc., 158, B416 (2011).

22. R. Nozaki, Y. Tabe, T. Chikahisa, and T. Tanuma, ECS Trans., 80(8), 335 (2017).

23. K. O'Neil, J. P. Meyers, R. M. Darling, and M. L. Perry, Int. J. Hydrogen Energy, 37, 373 (2012) 